\title{
Risk stratification and prognostic evaluation of endothelial cell-specific molecule1, von Willebrand factor, and a disintegrin-like and metalloprotease with thrombospondin type 1 motif for sepsis in the emergency department: An observational study
}

\author{
QING ZHANG ${ }^{1}$ and CHUN-SHENG $\mathrm{LI}^{2}$ \\ ${ }^{1}$ Department of Emergency, Beijing Chao-Yang Hospital, Capital Medical University; \\ ${ }^{2}$ Beijing Key Laboratory of Cardiopulmonary Cerebral Resuscitation, Beijing 100020, P.R. China
}

Received November 7, 2018; Accepted February 21, 2019

DOI: $10.3892 /$ etm.2019.7485

\begin{abstract}
This study evaluated endothelial cell-specific molecule1 (endocan), von Willebrand factor (vWF), and A disintegrin-like and metalloprotease with thrombospondin type 1 motif (ADAMTS-13), alone or in combination, in the risk stratification and prognosis prediction of patients with sepsis. Clinical data of 301 patients were prospectively analyzed, and divided into systemic inflammatory reaction syndrome, sepsis, severe sepsis, and septic shock groups. A total of 40 healthy individuals were studied as the control group. Endocan, vWF, ADAMTS-13, vWF/ADAMTS-13, and procalcitonin levels were measured, and Acute Physiology and Chronic Health Evaluation (APACHE II) score, Mortality in Emergency Department Sepsis (MEDS) score as well as Sequential Organ Failure Assessment (SOFA) score were calculated. The all-cause death or survival of each patient was recorded during the 28-day follow-up. The endocan, $\mathrm{vWF}$, and vWF/ADAMTS-13 levels significantly increased in patients and were positively correlated with disease severity. On the first day of admission, MEDS score, ADAMTS-13, and $\mathrm{vWF} / \mathrm{AMAMTS}-13$ ratio were independent predictors for 28-day mortality from sepsis. Moreover, the combination of $\mathrm{vWF} / A D A M T S-13$ ratio with MEDS score improved the accuracy in predicting the 28-day mortality from sepsis. On day 5, endocan, vWF, ADAMTS-13, and vWF/ADAMTS-13 ratio were independent predictors for the 28-day mortality from sepsis, while the combined use of endocan and
\end{abstract}

Correspondence to: Dr Chun-Sheng Li, Beijing Key Laboratory of Cardiopulmonary Cerebral Resuscitation, 8 Workers' Stadium South Road, Beijing 100020, P.R. China

E-mail: 1cscyyy@163.com

Key words: sepsis biomarkers, risk stratification, endothelial cell-specificmolecule, von Willebrand factor, Adisintegrin-like and metalloprotease with thrombospondintype1 motif
vWF/ADAMTS-13 ratio improved the prognostic value of individual indicators. Endocan, vWF, ADAMTS-13, and vWF/ADAMTS-13 ratio are valuable in the risk stratification and prognostic evaluation of sepsis as novel biomarkers.

\section{Introduction}

Sepsis is extremely common in intensive care units (ICUs) and emergency departments, with rising morbidity and mortality annually. Since sepsis is now recognized as one of the most challenging problems in critical care medicine, its rapid and early diagnosis and treatment have become particularly important $(1,2)$. Sepsis is mainly characterized by microcirculatory dysfunction, and its pathophysiology can be divided into two parts: Vascular endothelial cell dysfunction, and clot formation caused by a hypercoagulative state. Vascular endothelial cells play key roles in sepsis and sepsis-induced multiple organ dysfunction syndrome (MODS) because the vascular endothelial system, especially microvascular endothelium is mainly attacked during sepsis. The progression of sepsis can be indirectly reflected by the extent of endothelial cell injury and the degree of microthrombosis. Thus for the risk stratification and prognosis prediction, the investigation of markers for vascular endothelial cell damage is highly required (3).

Endothelial cell-specific molecule1 (endocan), the von Willebrand factor (vWF), and a disintegrin-like and metalloprotease with thrombospondin type 1 motif (ADAMTS-13) are three vascular endothelial cell markers to indicate endothelial damage and dysfunction. In this study, we measured the circulating levels of endocan, vWF, ADAMTS-13, and vWF/ADAMTS-13 in septic patients and explored the relationships of these indicators with sepsis severity and prognosis. Furthermore, the application of these markers during sepsis was elucidated.

\section{Subjects and methods}

Patients. The clinical data of 301 patients with systemic inflammatory reaction syndrome (SIRS) or sepsis, according to 
ACCP/SCCM criteria (4), who were treated in the Emergency Department in Beijing Chao-Yang Hospital (Beijing, China) from October 2014 to October 2015, were analyzed. According to the diagnostic criteria, these patients were divided into SIRS, sepsis, severe sepsis, and septic shock groups. Additionally, 40 healthy individuals in our center during the same period, were selected as the healthy control group.

Exclusion criteria were: i) Patients younger than 18 years, ii) patients or family members who refused to participate in the study, iii) patients with psychiatric disorders, iv) patients with tumors, v) patients who had undergone organ transplantation or who exhibited long-term use of immunosuppressive agents, vi) patients with allergic reactions, and vii) patients with dysfunction of two or more organs before the onset of the disease.

This study was approved by the Ethics Comittee of Institutional Review Board of Beijing Chao-Yang Hospital, Capital Medical University (Beijing, China). Patients who participated in this research had complete clinical data. The signed informed consents were obtained from the patients or the guardians.

Methods. Blood samples were collected from the elbow vein at baseline and 5 days later. The samples were placed in non-additive tubes, and centrifuged at 4,000 x $\mathrm{g}$ for $5 \mathrm{~min}$ at $4^{\circ} \mathrm{C}$. The serum was collected in $1.5 \mathrm{ml}$ centrifugal tubes, and then stored at $-80^{\circ} \mathrm{C}$ for further analysis. Endocan, vWF and ADAMTS-13 (SEC463Hu, CEA833Hu and SEA950Hu; Cloud-Clone Corp., Katy, TX, USA) concentrations were measured by the double-antibody sandwich enzyme-linked immunosorbent assay (DAS-ELISA). Procalcitonin (PCT) was measured by the MINI VIDAS ${ }^{\circledR}$ (Block Scientific Inc., New York, USA) fully automated analyzer. The patients' past histories, vital signs, routine test results, and radiographic examination findings were recorded. Baseline data were used for scoring on the following assessments: Acute Physiology and Chronic Health Evaluation (APACHE II) (5), Mortality in Emergency Department Sepsis (MEDS) (6), and Sequential Organ Failure Assessment (SOFA) (7). The outcome on the 28 th day after enrollment was used as the endpoint. During the follow-up, non-survivors were defined as patients who died of various reasons and survivors were defined as those who remained alive.

Statistical analysis. All statistical analysis was performed using SPSS 19.0 software (SPSS, Inc., Chicago, IL, USA). Normally distributed data were expressed as mean \pm standard deviations, and non-normally distributed data were expressed as medians (25-75\% interquartile ranges). Comparisons of measurement data between two or more groups were performed using one-way analysis of variance, whereas paired comparisons were performed using Least Significant Difference-t test. For non-normally distributed data, comparisons of the medians between two groups were performed using non-parametric tests, Kruskal-Wallis method. The test level $\alpha^{\prime}$ $\left(\alpha_{0.05}^{\prime}=0.05 /\right.$ number of paired comparisons; $\alpha_{0.01}^{\prime}=0.01 /$ number of paired comparisons) was adjusted according to the number of paired comparisons; a P-value of $<\alpha^{\prime}$ was regarded as statistically significant. The count data were compared using the Chi-square test. The independent predictive factors of the prognostic indicators were determined by binary logical regression analysis. The diagnostic and prognostic values of each indicator were evaluated using the receiver operating characteristic (ROC) curves. The area under the curve (AUC) was compared by:

$$
\mathrm{Z}=\left(\mathrm{A}_{1}-\mathrm{A}_{2}\right) / \sqrt{\mathrm{SE}_{1}^{2}+\mathrm{SE}_{2}^{2}},
$$

test criterion: $Z_{0.05}=1.96, Z_{0.01}=2.58, Z>Z_{0.05}$, then $\mathrm{P}<0.05$. $\mathrm{P}<0.05$ was considered to indicate a statistically significant difference.

\section{Results}

General characteristics of patients. Patients in all groups were comparable in terms of age, sex, previous diseases, and infection sites. The 28-day mortality, PCT level, MEDS score, APACHE II score, and SOFA score increased progressively in patients with sepsis (lowest), severe sepsis, or septic shock (highest) with significant difference $(\mathrm{P}<0.001$; Table I).

Levels of all biomarkers in each group. The levels of endocan and vWF, as well as vWF/ADAMTS-13 ratio progressively increased in the control (lowest), SIRS, sepsis, severe sepsis, and septic shock (highest) groups. However, the ADAMTS-13 level gradually decreased. The differences among these groups were statistically significant $(\mathrm{P}<0.05$; Table II).

Levels of all biomarkers in survivors and non-survivors. The levels of endocan and vWF, as well as vWF/ADAMTS-13 ratio were significantly higher in non-survivors than survivors on days 1 and $5(\mathrm{P}<0.01)$. However, ADAMTS-13 levels significantly decreased in non-survivors $(\mathrm{P}<0.01)$. Furthermore, levels of endocan and vWF, as well asvWF/ADAMTS-13 ratio were significantly lower on day 5 than day 1 in survivors (all $\mathrm{P}<0.01)$, whereas ADAMTS-13 levels significantly increased $(\mathrm{P}<0.01)$. In addition, levels of endocan and $\mathrm{vWF}$, as well as vWF/ADAMTS-13 ratio significantly increased on day 5 in non-survivors $(\mathrm{P}<0.05)$, whereas ADAMTS-13 significantly decreased over the same period $(\mathrm{P}<0.01$; Table III).

The disease condition was more critical in non-survivors than survivors. In the survivors, the levels of endocan and vWF, as well as vWF/ADAMTS-13 ratio decreased after 4 days of treatment, while the ADAMTS-13 levels increased, suggesting an improvement in disease condition. The changes of biomarker levels were reversed in the non-survivors.

Independent predictors of 28-day mortality in septic patients. On day 1, ADAMTS-13 levels, vWF/ADAMTS-13 ratio, and MEDS score were independent predictors of 28-day mortality for sepsis.

Logistic regression analysis was performed using the levels of endocan, vWF, ADAMTS-13, and vWF/ADAMTS-13 ratio, as well as the MEDS scores, on day 1 of admission. Since vWF/ADAMTS-13 is the ratio of VWF to ADAMTS-13, the input of all three indicators into the equation might influence each other, thus, vWF, ADAMTS-13, and vWF/ADAMTS-13 values were separately input into the equation, and the other indicators were kept unchanged. This produced two probability equations that enabled identification of ADAMTS-13 level, 
Table I. Patient characteristics.

\begin{tabular}{|c|c|c|c|c|c|c|}
\hline Variables & Control & SIRS & Sepsis & Severe sepsis & Septic shock & P-value \\
\hline Number & 40 & 40 & 77 & 132 & 52 & \\
\hline Age, years & 66.5 (26 to 82 ) & $65(14$ to 85$)$ & 65 (20 to 93$)$ & $69(26$ to 90$)$ & 67 (23 to 94$)$ & 0.602 \\
\hline Male n (\%) & 55.0 & 63.6 & 55.8 & 61.4 & 65.4 & 0.758 \\
\hline \multicolumn{7}{|l|}{ Infection site (n) } \\
\hline Lung & & & $54(70.1 \%)$ & $88(66.7 \%)$ & $36(69.2 \%)$ & 0.860 \\
\hline Abdominal cavity & & & $3(3.9 \%)$ & $6(4.5 \%)$ & $4(7.7 \%)$ & 0.650 \\
\hline Urinary system & & & $1(1.3 \%)$ & $7(5.3 \%)$ & $2(3.8 \%)$ & 0.430 \\
\hline Gastrointestinal tract & & & $7(9.1 \%)$ & $7(5.3 \%)$ & $2(3.8 \%)$ & 0.486 \\
\hline Skin and soft tissue & & & $2(2.6 \%)$ & $5(3.8 \%)$ & $3(5.8 \%)$ & 0.635 \\
\hline Central system & & & $3(3.9 \%)$ & $5(3.8 \%)$ & $1(1.9 \%)$ & 0.911 \\
\hline Hepatobiliary system & & & $7(9.1 \%)$ & $14(10.6 \%)$ & $4(7.7 \%)$ & 0.821 \\
\hline \multicolumn{7}{|c|}{ Previous disease history n (\%) } \\
\hline COPD & & $12(30.0 \%)$ & $27(35.1 \%)$ & $51(38.6 \%)$ & $16(30.8 \%)$ & 0.658 \\
\hline Cardiovascular diseases & & $13(32.5 \%)$ & $29(37.7 \%)$ & $53(40.2 \%)$ & $17(32.7 \%)$ & 0.659 \\
\hline Cerebrovascular diseases & & $9(22.5 \%)$ & $19(24.7 \%)$ & $39(29.6 \%)$ & $20(38.4 \%)$ & 0.283 \\
\hline Diabetes & & $11(27.5 \%)$ & $27(35.1 \%)$ & $49(37.1 \%)$ & $21(40.4 \%)$ & 0.617 \\
\hline Others & & $4(10.0 \%)$ & $12(15.6 \%)$ & $25(18.9 \%)$ & $10(19.2 \%)$ & 0.564 \\
\hline 28-day mortality n (\%) & & $1(2.5 \%)$ & $16(20.8 \%)$ & $43(32.6 \%)$ & $36(69.2 \%)$ & $<0.001$ \\
\hline PCT (ng/l) & & $0.05(0.05-0.43)$ & $2.59(0.41-5.09)$ & $3.97(1.04-8.06)$ & $9.27(6.84-13.30)$ & ) $<0.001$ \\
\hline MEDS score & & $8.0(5.5-10.5)$ & $9.0(7.0-11.0)$ & $11.0(8.0-14.0)$ & $14.5(12.0-16.0)$ & $<0.001$ \\
\hline APACHE II score & & $9.1 \pm 3.6$ & $12.0 \pm 5.2$ & $16.2 \pm 6.6$ & $22.9 \pm 6.7$ & $<0.001$ \\
\hline SOFA score & & $2.0(1.0-2.5)$ & $3.0(2.0-6.0)$ & $7.5(5.0-9.0)$ & $11.0(10.0-14.0)$ & $<0.001$ \\
\hline
\end{tabular}

COPD, chronic obstructive pulmonary diseases; PCT, procalcitonin; MEDS, mortality in emergency department sepsis; APACHE II, acute physiology and chronic health evaluation; SOFA, sequential organ failure assessment.

Table II. Levels of all biomarkers in each group.

\begin{tabular}{|c|c|c|c|c|c|c|}
\hline Items & Control & SIRS & Sepsis & Severe sepsis & Septic shock & P-value \\
\hline Number & 40 & 40 & 77 & 132 & 52 & \\
\hline $\begin{array}{l}\text { Endocan } \\
(\mathrm{ng} / \mathrm{ml})\end{array}$ & $32.53 \pm 9.13$ & $47.31 \pm 6.11$ & $52.86 \pm 5.17$ & $54.99 \pm 5.28^{a}$ & $58.25 \pm 4.08$ & $<0.001$ \\
\hline vWF (ng/l) & $1729.49 \pm 565.60$ & $2736.22 \pm 436.98$ & $3199.17 \pm 425.61$ & $3909.27 \pm 334.02$ & $4161.97 \pm 275.65$ & $<0.001$ \\
\hline $\begin{array}{l}\text { ADAMTS-13 } \\
(\mathrm{pg} / \mathrm{ml})\end{array}$ & $328.06 \pm 49.22$ & $268.33 \pm 24.64$ & $238.38 \pm 25.96$ & $215.44 \pm 21.89$ & $199.10 \pm 14.66$ & $<0.001$ \\
\hline vWF/ADAMTS1 & $5.43 \pm 2.06$ & $10.32 \pm 2.14$ & $13.59 \pm 2.40$ & $18.34 \pm 2.54$ & $21.01 \pm 2.01$ & $<0.001$ \\
\hline
\end{tabular}

Endocan, endothelial cell specific molecule-1; vWF, von Willebrand factor; ADAMTS-13, a disintegrin-like and metalloprotease with thrombospondin type 1 motif. ${ }^{\mathrm{P}}<0.05$ vs sepsis group.

vWF/ADAMTS-13 ratio, and MEDS score as independent predictors of the 28-day mortality from sepsis on day 1 of admission. The equation (i):

$$
\left(\mathrm{P}=1 /\left[1+\mathrm{e}^{-(-9.875+0.290 \times \text { MEDS }+0.239 \times \text { vWF/ADAMTS }-13)}\right]\right)
$$

was obtained by logistic regression analyses involving endocan, vWF/ADAMTS-13 ratio, and MEDS score. The equation (ii) $\mathrm{P}=1 /\left[1+\mathrm{e}^{-(0.296 \times \text { MEDS }-0.029 \times \text { ADAMTS }-13)}\right]$ was obtained by logistic regression analyses involving endocan, $\mathrm{vWF}$, and ADAMTS-13 levels, as well as MEDS score (Tables IV and V).
On the fifth day of admission, levels of endocan, vWF and ADAMTS-13, as well as vWF/ADAMTS-13 ratio were independent predictors of 28-day mortality for sepsis.

Logistic regression analysis was performed on the endocan, vWF and ADAMTS-13 levels, as well as the vWF/ADAMTS-13 ratio, on day 5 of admission. The vWF, ADAMTS-13, and vWF/ADAMTS-13 values were separately input into the equation, with other indicators unchanged. Therefore, two probability equations were obtained: 
Table III. Comparisons of biomarkers between survivors and non-survivors on days 1 and 5 after admission.

\begin{tabular}{|c|c|c|c|}
\hline Variables & Survivors & Non-survivors & P-value \\
\hline Number & 205 & 96 & \\
\hline \multicolumn{4}{|l|}{ Endocan (ng/ml) } \\
\hline Day 1 & $53.14 \pm 6.55$ & $56.22 \pm 3.71$ & $<0.01$ \\
\hline Day 5 & $46.29 \pm 6.84$ & $57.73 \pm 8.16$ & $<0.01$ \\
\hline P-value & $<0.01$ & 0.049 & \\
\hline Difference $(\%)$ & -12.9 & +2.7 & \\
\hline \multicolumn{4}{|l|}{ vWF (ng/l) } \\
\hline Day 1 & $3476.83 \pm 586.27$ & $3965.26 \pm 468.88$ & $<0.01$ \\
\hline Day 5 & $3296.10 \pm 557.98$ & $4052.11 \pm 482.55$ & $<0.01$ \\
\hline P-value & $<0.01$ & 0.002 & \\
\hline Difference $(\%)$ & -5.2 & +2.2 & \\
\hline \multicolumn{4}{|c|}{ ADAMTS-13 (pg/ml) } \\
\hline Day 1 & $233.05 \pm 31.03$ & $206.86 \pm 18.64$ & $<0.01$ \\
\hline Day 5 & $247.32 \pm 33.91$ & $199.99 \pm 21.45$ & $<0.01$ \\
\hline P-value & $<0.01$ & $<0.01$ & \\
\hline Difference $(\%)$ & +6.1 & -3.3 & \\
\hline \multicolumn{4}{|c|}{ vWF/ADAMTS-13 } \\
\hline Day 1 & $15.35 \pm 3.90$ & $19.39 \pm 3.22$ & $<0.01$ \\
\hline Day 5 & $13.73 \pm 3.60$ & $20.61 \pm 3.93$ & $<0.01$ \\
\hline P-value & $<0.01$ & $<0.01$ & \\
\hline Difference (\%) & -10.6 & +6.3 & \\
\hline
\end{tabular}

Endocan, endothelial cell specific molecule-1; vWF, von Willebrand factor; ADAMTS-13, a disintegrin-like and metalloprotease with thrombospondin type 1 motif.

Table IV. Independent predictors of 28-day mortality in septic patients on day 1 of admission-equation (i).

\begin{tabular}{|c|c|c|c|c|c|c|c|}
\hline \multirow{2}{*}{$\begin{array}{l}\text { Independent } \\
\text { predictors }\end{array}$} & \multirow[b]{2}{*}{$\mathrm{B}$} & \multirow[b]{2}{*}{ SE } & \multirow[b]{2}{*}{ Wald } & \multirow[b]{2}{*}{ P-value } & \multirow[b]{2}{*}{$\operatorname{Exp}(B)$} & \\
\hline & & & & & & $5 \%$ & $95 \%$ \\
\hline MEDS & 0.290 & 0.049 & 35.325 & $<0.001$ & 1.337 & 0.199 & 0.412 \\
\hline vWF/ ADAMTS-13 & 0.239 & 0.050 & 23.179 & $<0.001$ & 1.269 & 0.147 & 0.362 \\
\hline Constant & -9.875 & 1.775 & 30.952 & $<0.001$ & 0.000 & & \\
\hline
\end{tabular}

SE, standard error; CI, confidence interval; MEDS, mortality in emergency department sepsis; vWF, von Willebrand factor; ADAMTS-13, a disintegrin-like and metalloprotease with thrombospondin type 1 motif.

equation (iii) $\mathrm{P}=1 /\left[1+\mathrm{e}^{-(-12.622+0.106 \times \text { endocan }+0.380 \times \text { vWF/ADAMTS }-13)}\right]$ was obtained by logistic regression analyses involving endocan level and vWF/ADAMTS-13 ratio. Equation (iv):

$$
\left.\mathrm{P}=1 /\left[1+\mathrm{e}^{-(0.106} \mathrm{x} \text { endocan }+0.001 \times \mathrm{vWF}-0.042 \mathrm{x} \text { ADAMTS }-13\right)\right]
$$

was obtained by logistic regression analyses involving endocan, vWF and ADAMTS-13 levels. On the fifth day of admission, endocan, vWF and ADAMTS-13 levels, as well as vWF/ADAMTS-13 ratio, were independent predictors of the 28-day mortality (Tables VI and VII).

ROC curves of the independent predictors of 28-day mortality in septic patients. Fig. 1 shows the ROC curves of endocan, vWF, ADAMTS-13, vWF/ADAMTS-13 ratio, and MEDS score in predicting the 28-day mortality rate of sepsis on day 1 of admission. Table VIII shows the area under the ROC curve (AUC) and 95\% confidence interval (95\% CI) for each indicator. The MEDS score exhibited the highest AUC (0.809) among all the indicators, which was significantly higher than the AUC $(0.656)$ of endocan $(\mathrm{P}<0.01)$, although it showed no significant difference when comparing with the AUCs of other indicators. The combination of vWF/ADAMTS-13 ratio with MEDS score (AUC 0.856) remarkably increased the prognostic value, especially when comparing with endocan $(0.656, \mathrm{P}<0.01)$, $\operatorname{vWF}(0.751, \mathrm{P}<0.01)$, or ADAMTS-13 (0.761, $\mathrm{P}<0.05)$ alone.

In Table IX, the results are shown of the assessment of the prognostic capabilities of all indicators in septic patients. The 
Table V. Independent predictors of 28-day mortality in septic patients on day 1 of admission-equation (ii).

$95 \% \mathrm{CI}$

\begin{tabular}{lccccccc}
$\begin{array}{l}\text { Independent } \\
\text { predictors }\end{array}$ & $\mathrm{B}$ & $\mathrm{SE}$ & Wald & P-value & Exp (B) & $5 \%$ & $95 \%$ \\
\hline MEDS & 0.296 & 0.050 & 35.032 & $<0.001$ & 1.345 & 0.206 & 0.432 \\
ADAMTS-13 & -0.029 & 0.008 & 13.053 & $<0.001$ & 0.972 & -0.047 & -0.014 \\
\hline
\end{tabular}

SE, standard error; CI, confidence interval; MEDS, mortality in emergency department sepsis; ADAMTS-13, a disintegrin-like and metalloprotease with thrombospondin type 1 motif.

Table VI. Independent predictors of 28-day mortality in septic patients on day 5 of admission-equation (iii).

\begin{tabular}{|c|c|c|c|c|c|c|c|}
\hline \multirow{2}{*}{$\begin{array}{l}\text { Independent } \\
\text { predictors }\end{array}$} & \multirow[b]{2}{*}{$\mathrm{B}$} & \multirow[b]{2}{*}{$\mathrm{SE}$} & \multirow[b]{2}{*}{ Wald } & \multirow[b]{2}{*}{ P-value } & \multirow[b]{2}{*}{$\operatorname{Exp}(\mathrm{B})$} & \multicolumn{2}{|c|}{$95 \% \mathrm{CI}$} \\
\hline & & & & & & $5 \%$ & $95 \%$ \\
\hline Endocan & 0.106 & 0.028 & 14.353 & $<0.001$ & 1.112 & 0.056 & 0.174 \\
\hline vWF/ADAMTS 13 & 0.380 & 0.063 & 36.031 & $<0.001$ & 1.463 & 0.265 & 0.549 \\
\hline Constant & -12.622 & 1.538 & 67.343 & $<0.001$ & 0.000 & & \\
\hline
\end{tabular}

SE, standard error; CI, confidence interval; endocan, endothelial cell specific molecule-1; vWF, von Willebrand factor; ADAMTS-13, a disintegrin-like and metalloprotease with thrombospondin type 1 motif.

Table VII. Independent predictors of 28-day mortality in septic patients on day 5 of admission-equation (iv).

\begin{tabular}{lcccccrr}
\hline $\begin{array}{l}\text { Independent } \\
\text { predictors }\end{array}$ & $\mathrm{B}$ & $\mathrm{SE}$ & Wald & P-value & Exp (B) & $5 \%$ & \multicolumn{2}{c}{$95 \% \mathrm{CI}$} \\
\hline Endocan & 0.106 & 0.028 & 14.499 & $<0.001$ & 1.111 & 0.058 & 0.168 \\
vWF & 0.001 & 0.000 & 7.563 & 0.006 & 1.001 & 0.000 & 0.002 \\
ADAMTS-13 & -0.042 & 0.009 & 23.682 & $<0.001$ & 0.958 & -0.065 & -0.028 \\
\hline
\end{tabular}

SE, standard error; CI, confidence interval; endocan, endothelial cell specific molecule-1; vWF: von Willebrand factor; ADAMTS-13, a disintegrin-like and metalloprotease with thrombospondin type 1 motif.

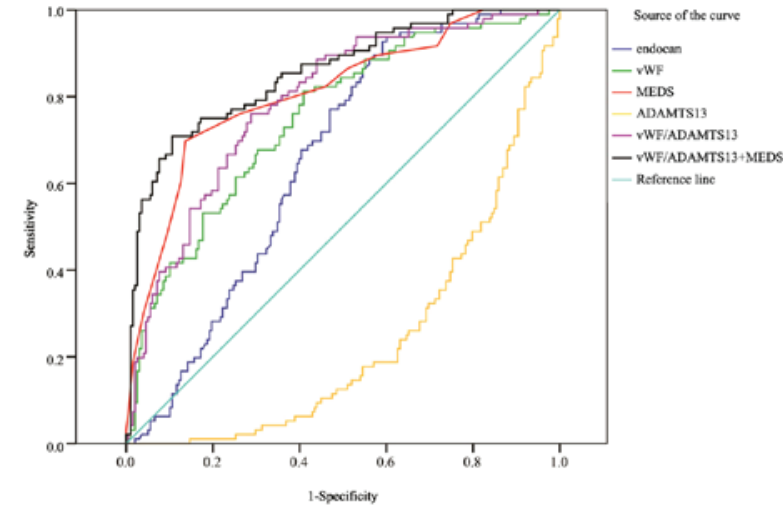

Figure 1. ROC curves of all indicators in predicting the 28-day mortality on day 1 . ROC, receiver operating characteristic.

combination of vWF/ADAMTS-13 ratio with MEDS score exhibited superior sensitivity (70.8), specificity (89.4), positive predictive value (PPV) (76.4), and negative predictive value (NPV) (86.3), compared with individual indicators.

Fig. 2 shows the ROC curves of endocan,vWF,ADAMTS-13, and $\mathrm{vWF} / \mathrm{ADAMTS}-13$ ratio in predicting the 28-day mortality rate of sepsis on the fifth day of admission. The AUC (0.905) of the vWF/ADAMTS-13 ratio was the highest among all indicators, but without significant difference when comparing with the AUCs of other biomarkers. The combination of vWF/ADAMTS-13 ratio with endocan (AUC 0.921) remarkably increased the prognostic value, especially when comparing with endocan $(0.853, \mathrm{P}<0.05)$ or $\operatorname{vWF}(0.850, \mathrm{P}<0.05)$ alone. The predictive value of vWF/ADAMTS-13 + endocan (AUC 0.921) on day 5 was significantly higher than that of vWF/ADAMTS-13 + MEDS score (AUC 0.856, $\mathrm{P}<0.05$ ) on day 1 (Table $\mathrm{X}$ ). The combination of endocan level+vWF/ADAMTS-13 ratio exhibited superior sensitivity (93.8), specificity (79.3), PPV (68.7), and NPV (96.3), compared with individual indicators (Table XI). 
Table VIII. Area under ROC curve (AUC) of all indicators in predicting the 28-day mortality from sepsis on day 1 of admission.

\begin{tabular}{lccccr}
\hline & & & & \multicolumn{2}{c}{$95 \%$ CI } \\
\cline { 4 - 6 } Predictors & AUC & SE & P & $5 \%$ & $95 \%$ \\
\hline Endocan & $0.656^{\mathrm{a}}$ & 0.031 & $<0.001$ & 0.595 & 0.717 \\
vWF & $0.751^{\mathrm{a}}$ & 0.030 & $<0.001$ & 0.693 & 0.810 \\
ADAMTS-13 & $0.761^{\mathrm{b}}$ & 0.028 & $<0.001$ & 0.706 & 0.815 \\
vWF/ADAMTS-13 & 0.790 & 0.027 & $<0.001$ & 0.737 & 0.844 \\
MEDS & 0.809 & 0.028 & $<0.001$ & 0.755 & 0.863 \\
vWF/ADAMTS-13 +MEDS & 0.856 & 0.024 & $<0.001$ & 0.808 & 0.903 \\
\hline
\end{tabular}

AUC, area under the receiver operating characteristic curve; SE, standard error; CI, confidence interval; endocan: endothelial cell specific molecule-1; vWF, von Willebrand factor; ADAMTS-13, a disintegrin-like and metalloprotease with thrombospondin type 1 motif; MEDS, mortality in emergency department sepsis. ${ }^{\mathrm{a}} \mathrm{P}<0.01$; ${ }^{\mathrm{b}} \mathrm{P}<0.05$ vs vWF/ADAMTS-13 + MEDS.

Table IX. The cut-off value of each indicator for the prognosis of sepsis and the relevant evaluation results on day 1 of admission.

\begin{tabular}{|c|c|c|c|c|c|}
\hline Prognostic factors & Cut-off value & Sensitivity (\%) & Specificity (\%) & PPV $(\%)$ & $\operatorname{NPV}(\%)$ \\
\hline Endocan (ng/ml) & 51.84 & 92.7 & 40.9 & 43.2 & 92.0 \\
\hline vWF (ng/l) & 3674.67 & 81.3 & 59.1 & 49.1 & 86.7 \\
\hline ADAMTS-13 (pg/ml) & 221.58 & 62.6 & 81.3 & 61.9 & 81.7 \\
\hline vWF/ADAMTS-13 & 17.51 & 76.0 & 71.2 & 56.1 & 86.0 \\
\hline MEDS score & 12.5 & 69.8 & 86.4 & 71.3 & 85.5 \\
\hline vWF/ADAMTS-13 + MEDS & 0.47 & 70.8 & 89.4 & 76.4 & 86.3 \\
\hline
\end{tabular}

PPV, positive predictive value; NPV, negative predictive value; endocan, endothelial cell specific molecule-1; vWF, von Willebrand factor; ADAMTS-13, a disintegrin-like and metalloprotease with thrombospondin type 1 motif; MEDS, mortality in emergency department sepsis.

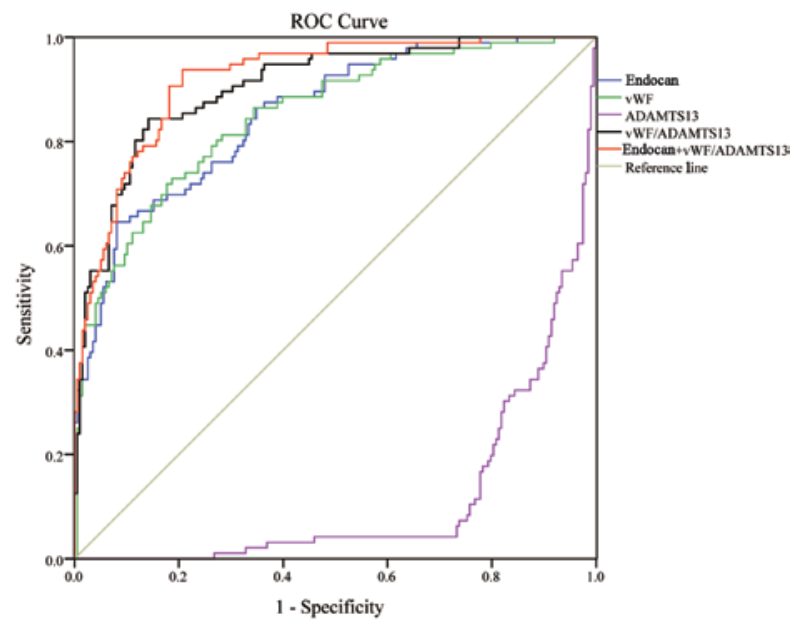

Figure 2. ROC curves of all indicators in predicting the 28-day mortality on day 5. ROC, receiver operating characteristic.

\section{Discussion}

As one of the leading causes of death in critically ill patients, studies have shown that the case-fatality rate in patients with severe sepsis or septic shock reached 20-54\% (5,8-11). Despite the application of more advanced life support and new generations of antibiotics in recent years, the number of annual deaths from sepsis continues to increase. Furthermore, the fatality rate increases by $5-10 \%$ for each hour when the appropriate antibiotic treatment is delayed $(12,13)$. Therefore, the early diagnosis and treatment of sepsis are both particularly important. Biomarkers can increase the accuracy of diagnosis and help to monitor the infection process. In the absence of typical clinical symptoms, validated biomarkers can objectively reflect the severity of the disease and furthermore monitor the pathophysiological process of the disease, as well as the response to treatment interventions (14). Thereafter, it is essential to search for biomarkers that are valuable for early diagnosis, accurate stratification, sensitive therapeutic monitoring, and precise prediction of prognosis.

In this study, the levels of endocan and vWF, as well as $v W F / A D A M T S-13$ ratio progressively increased in the healthy control (lowest), SIRS, sepsis, severe sepsis, and septic shock (highest) groups, whereas ADAMTS-13 progressively decreased, with statistically significant difference, suggesting that these biomarkers may play specific roles in risk stratification. Importantly, patients with more severe disease conditions exhibited higher endocan, vWF, and vWF/ADAMTS-13 ratio levels, and lower ADAMTS-13 levels. Because vascular endothelial injury increases with severity rate of sepsis, the endothelial cells become more active, followed by the occurrence of MODS. Therefore, these combined biomarkers not only indicate disease severity but 
Table X. Area under ROC curve (AUC) of all indicators in predicting the 28-day mortality from sepsis on day 5.

\begin{tabular}{|c|c|c|c|c|c|}
\hline \multirow[b]{2}{*}{ Predictors } & \multirow[b]{2}{*}{ AUC } & \multirow[b]{2}{*}{$\mathrm{SE}$} & \multirow[b]{2}{*}{ P-value } & \multicolumn{2}{|c|}{$95 \% \mathrm{CI}$} \\
\hline & & & & $5 \%$ & $95 \%$ \\
\hline vWF & $0.850^{\mathrm{a}}$ & 0.024 & $<0.001$ & 0.803 & 0.897 \\
\hline Endocan & $0.853^{\mathrm{a}}$ & 0.023 & $<0.001$ & 0.807 & 0.898 \\
\hline ADAMTS-13 & 0.886 & 0.020 & $<0.001$ & 0.847 & 0.924 \\
\hline vWF/ADAMTS-13 & 0.905 & 0.019 & $<0.001$ & 0.868 & 0.941 \\
\hline Endocan + vWF/ADAMTS-13 & 0.921 & 0.016 & $<0.001$ & 0.889 & 0.952 \\
\hline
\end{tabular}

AUC, area under the receiver operating characteristic curve; SE, standard error; CI, confidence interval; endocan, endothelial cell specific molecule-1; vWF, von Willebrand factor; ADAMTS-13, a disintegrin-like and metalloprotease with thrombospondin type 1 motif. ${ }^{\text {a }}<0.05$ vs endocan + vWF/ADAMTS-13.

Table XI. The cut-off value of each indicator for the prognosis of sepsis and the relevant evaluation results on day 5.

\begin{tabular}{|c|c|c|c|c|c|}
\hline Prognostic factors & Cut-off value & Sensitivity (\%) & Specificity (\%) & PPV $(\%)$ & $\operatorname{NPV}(\%)$ \\
\hline Endocan (ng/ml) & 55.89 & 64.6 & 91.9 & 79.5 & 84.3 \\
\hline vWF (ng/l) & 3814.84 & 71.9 & 82.3 & 66.3 & 85.8 \\
\hline ADAMTS-13 (pg/ml) & 229.19 & 73.2 & 95.8 & 89.7 & 88.0 \\
\hline vWF/ADAMTS-13 & 16.93 & 84.4 & 85.9 & 74.3 & 91.9 \\
\hline Endocan + vWF/ADAMTS-13 & 0.22 & 93.8 & 79.3 & 68.7 & 96.3 \\
\hline
\end{tabular}

PPV, positive predictive value; NPV, negative predictive value; endothelial cell specific molecule-1; vWF, von Willebrand factor; ADAMTS-13, a disintegrin-like and metalloprotease with thrombospondin type 1 motif.

may serve as independent indicators of organ dysfunction and poor prognosis $(15,16)$.

Based on our results, MEDS scoring might be preferentially on day 1 to predict the likelihood of death. If patients survive to day 5, the vWF/ADAMTS-13 ratio would be a more feasible biomarker to predict death risk. Furthermore, comparisons of AUC have shown that the AUCs of the combined vWF/ADAMTS-13 ratio were higher than those of vWF or ADAMTS-13 alone on both days 1 and 5, suggesting that vWF/ADAMTS-13 ratio is superior to vWF or ADAMTS-13 alone in both determining disease severity and predicting prognosis. Claus et al (17) found that vWF/ADAMTS-13 ratio was associated with the severity and prognosis of organ failure in patients with systemic inflammation, and that this combined ratio was more valuable than vWF alone in decision-making, which is consistent to our finding. Finally, we also found that the combination of two indicators was superior to a single indicator: for septic patients in the emergency department, endocan + vWF/ADAMTS-13 was the most valuable indicator for predicting the 28-day fatality rate on day 5 .

Vascular endothelial cells play an important role in sepsis and sepsis-induced MODS. The progression of sepsis can be indirectly reflected by the extent of endothelial cell injury and the degree of microthrombus formation. Specifically secreted by endothelial cells, endocan is continuously released from cells when endothelial cells are damaged in a septic patient, thus mediating its physiological functions in regulating leukocyte adhesion and migration, as well as in preventing leukocytes from entering tissue and causing tissue damage. The serum level of endocan increased significantly and was correlated with severity of sepsis $(3,18)$.

In the pathogenesis of sepsis, vascular endothelial cells and platelet-derived vWF molecules form multimers that are much larger than those in normal plasma. These 'ultralarge' vWF (UL-vWF) multimers are connected with the P-selectin on the endothelial cell surface in a beaded chain fashion and bind to the glycoprotein $\mathrm{Ib}(\mathrm{GpIb})$ on the surface of circulating platelets. Other platelets are assembled around the UL-vWF via the activated glycoprotein IIb-IIIa (GPIIB-IIIA) complexes, forming large platelet thrombosis that may cause embolism. Once vascular endothelial cells are injured, UL-vWF cleaves from the surface of endothelial cells. These free UL-vWF-platelets can block downstream small blood vessels, resulting in ischemia of tissues/organs, ultimately causing MODS (19). The damage of vascular endothelial cells will inevitably lead to the massive release of vWF $(20,21)$. High vWF level, as an important biomarker of vascular endothelial damage, has been confirmed in sepsis $(15,17,22)$.

ADAMTS-13 is an enzyme that degrades vWF-platelet complexes, reducing thrombus formation (23), avoiding microvenous thrombosis, and regulating the thrombus reaction in the injured arteries (24). Increased vWF levels along with decreased ADAMTS-13 levels have been detected in septic patients (25). Martin et al (26) reported the formation of a 
large number of vWF polymers during sepsis, which consumed ADAMTS-13 in vivo. Additionally, sepsis was associated with the excessive production of interleukin-6, a proinflammatory cytokine, which reduced the speed of ADAMTS-13 in degrading $\mathrm{vWF}$ polymers. Abnormal secretion of ADAMTS-13 in septic patients may explain the concomitant decrease in ADAMTS-13 activity $(27,28)$. As ADAMTS-13 activity dramatically decreases, plasma vWF macromolecules cannot be degraded. Resultantly, more platelets bind to vWF to form small thrombi, which block the microvessels and aggravate microcirculation disorders $(15,24)$.

A sepsis biomarker should be able to identify either the onset of SIRS or compensated anti-inflammatory response syndrome (CARS) before the onset of MODS and aid in the lowering of mortality rates. However, because of the vague and broad definition of sepsis along with its various manifestations and severity levels, it is difficult to ascertain a definitive biomarker which could aid in therapeutic strategies. Currently, no biological molecular markers are used in the diagnosis or prognosis of sepsis in the United States (29).

Studies have reported the high specificity and sensitivity of C-reactive protein (CRP) in the sepsis diagnosis. However, other reports showed that CRP levels are not indicative of survival in sepsis patients (30). PCT has mixed value as a biomarker in the diagnosis and prognosis of sepsis. Elevations of PCT are not as specific for infection as was once believed, which may be elevated in a number of disorders in the absence of infection, especially following trauma (31-33). PCT levels may vary early during the development of sepsis and the test's predictive power is probably only significant later in the patient's course (34-35).

In this study, several biomarkers were evaluated to predict total in hospital mortality early in suspected sepsis, and we found that endocan, vWF and ADAMTS-13 levels, were easily and timely acquired for clinicians to rapidly diagnose and extend treatment beyond the standard therapy. Moreover, a combination of vWF/ADAMTS-13 ratio may be more effective. These biomarkers had the best predictive performance, which outperformed clinical criteria and other more extensively studied biomarkers (e.g. CRP and PCT) used for diagnosis and mortality prediction in the setting of sepsis. Our findings suggested that endocan, vWF and ADAMTS-13 levels, as well as vWF/ADAMTS-13 ratio, when combined with the clinical SIRS criteria that defined eligibility for enrollment, may provide valuable tool to predict mortality in sepsis.

In the present study there were also some limitations. As a single-center study, it was limited by its small sample size, and the findings need to be further validated in large-scale multiple-center studies. Second, systematic scoring was not performed on day 5 , which might compromise the conclusions of the analysis regarding the predictors of 28-day mortality from sepsis. Finally, while patients who had received treatment in other hospitals were excluded during enrollment, some subjects in our study might still have been treated with oral drugs for several days (on their own) before admission, which might have affected the measurement results of all indicators.

In conclusion, Endocan, vWF and ADAMTS-13 levels, as well as vWF/ADAMTS-13 ratio are valuable in the risk stratification and prognostic evaluation of sepsis, providing novel sepsis biomarkers in clinic.

\section{Acknowledgements}

The authors sincerely thank the ED staff, the Physical examination center of Beijing Chao-Yang Hospital, and the Biochemistry Laboratory staff for their helpful contributions to this study.

\section{Funding}

The authors have not received a specific grant for this research from any funding agency in the public, commercial or not-for-profit sectors.

\section{Availability of data and materials}

The datasets used and/or analyzed during the current study are available from the corresponding author on reasonable request.

\section{Authors' contributions}

CSL designed this study, QZ conducted the trial and collected and analyzed data, performed statistical analysis, drafted and wrote the manuscript. CSL takes responsibility for the paper as a whole. All authors read and approved the final manuscript.

\section{Ethics approval and consent to participate}

This study was approved by the Ethics Comittee of Institutional Review Board of Beijing Chao-Yang Hospital, Capital Medical University (Beijing, China). Patients who participated in this research had complete clinical data. The signed informed consents were obtained from the patients or the guardians

\section{Patient consent for publication}

Not applicable.

\section{Competing interests}

The authors declare that they have no competing interests.

\section{References}

1. Lever A and Mackenzie I: Sepsis: Definition, epidemiology, and diagnosis. BMJ 335: 879-883, 2007.

2. Zambon M, Ceola M, Almeida-de-Castro R, Gullo A and Vincent JL: Implementation of the surviving sepsis campaign guidelines for severe sepsis and septic shock: We could go faster. J Crit Care 23: 455-460, 2008.

3. Pierrakos C and Vincent JL: Sepsis biomarkers: A review. Crit Care 14: R15, 2010.

4. Levy MM, Fink MP, Marshall JC, Abraham E, Angus D, Cook D, Cohen J, Opal SM, Vincent JL and Ramsay G; SCCM/ESICM/ACCP/ATS/SIS: 2001 SCCM/ESICM/ACCP/ATS/SIS International Sepsis Definitions Conference. Crit Care Med 31: 1250-1256, 2003.

5. Knaus WA, Draper EA, Wagner DP and Zimmerman JE: APACHE II: A severity of disease classification system. Crit Care Med 13: 818-829, 1985

6. Shapiro NI, Wolfe RE, Moore RB, Smith E, Burdick E and Bates DW: Mortality in Emergency Department Sepsis (MEDS) score: a prospectively derived and validated clinical prediction rule. Crit Care Med 31: 670-675, 2003. 
7. Vincent JL, Moreno R, Takala J, Willatts S, De Mendonca A, Bruining H, Reinhart CK, Suter PM and Thijs LG: The SOFA (Sepsis-related Organ Failure Assessment) score to describe organ dysfunction/failure. On behalf of the working group on sepsis-related problems of the European Society of Intensive Care Medicine. Intensive Care Med 22: 707-710, 1996.

8. Shirakawa K, Naitou K, Hirose J, Takahashi T and Furusako S: Presepsin (sCD14-ST): Development and evaluation of one-step ELISA with a new standard that is similar to the form of presepsin in septic patients. Clin Chem Lab Med 49: 937-939, 2011.

9. Alberti C, Brun-Buisson C, Burchardi H, Martin C, Goodman S, Artigas A, Sicignano A, Palazzo M, Moreno R, Boulmé R, et al: Epidemiology of sepsis and infection in ICU patients from an international multicentre cohort study. Intensive Care Med 28: 108-121, 2002.

10. Vincent JL, Sakr Y, Sprung CL, Ranieri VM, Reinhart K, Gerlach H, Moreno R, Carlet J, Le Gall JR and Payen D; Sepsis occurrence in acutely Ill patients investigators: Sepsis in European intensive care units: Results of the SOAP study. Crit Care Med 34: 344-353, 2006.

11. Esteban A, Frutos-Vivar F, Ferguson ND, Peñuelas O, Lorente JA, Gordo F, Honrubia T, Algora A, Bustos A, García G, et al: Sepsis incidence and outcome: Contrasting the intensive care unit with the hospital ward. Crit Care Med 35: 1284-1289, 2007.

12. Gonsalves MD and Sakr Y: Early identification of sepsis. Curr Infect Dis Rep 12: 329-335, 2010.

13. Bauer M and Reinhart K: Molecular diagnostics of sepsis - where are we today? Int J Med Microbiol 300: 411-413, 2010.

14. Levy MM: Preface biomarkers in critical illness. Crit Care Clin 27: xiii-xv, 2011.

15. Bongers TN, de Maat MP, van Goor ML, Bhagwanbali V, van Vliet HH, Gómez García EB, Dippel DW and Leebeek FW: High von Willebrand factor levels increase the risk of first ischemic stroke: Influence of ADAMTS13, inflammation, and genetic variability. Stroke 37: 2672-2677, 2006.

16. Nguyen TC,Liu A, Liu L, Ball C, Choi H, May WS, Aboulfatova K, Bergeron AL and Dong JF: Acquired ADAMTS-13 deficiency in pediatric patients with severe sepsis. Haematologica 92: 121-124, 2007.

17. Claus RA, Bockmeyer CL, Budde U, Kentouche K, Sossdorf M, Hilberg T, Schneppenheim R, Reinhart K, Bauer M, Brunkhorst FM, et al: Variations in the ratio between von Willebrand factor and its cleaving protease during systemic inflammation and association with severity and prognosis of organ failure. Thromb Haemost 101: 239-247, 2009.

18. Scherpereel A, Depontieu F, Grigoriu B, Cavestri B, Tsicopoulos A, Gentina T, Jourdain M, Pugin J, Tonnel AB and Lassalle P: Endocan, a new endothelial marker in human sepsis. Crit Care Med 34: 532-537, 2006.

19. Chng WJ, Yip CY, Baliwag MB and Liu TC: Differential effect of the ABO blood group on von Willebrand factor collagen binding activity and ristocetin cofactor assay. Blood Coagu Fibrinolysis 16: 75-78, 2005.

20. Sadler JE: Biochemistry and genetics of von Willebrand factor. Annu Rev Biochem 67: 395-424, 1998.

21. van Mourik JA, Boertjes R, Huisveld IA, Fijnvandraat K, Pajkrt D, van Genderen PJ and Fijnheer R: von Willebrand factor propeptide in vascular disorders: a tool to distinguish between acute and chronic endothelial cell perturbation. Blood 94: $179-185,1999$.
22. Lerolle N, Dunois-Lardé C, Badirou I, Motto DG, Hill G, Bruneval P, Diehl JL, Denis CV and Baruch D: von Willebrand factor is a major determinant of ADAMTS-13 decrease during mouse sepsis induced by cecum ligation and puncture. J Thromb Haemost 7: 843-850, 2009.

23. Dong JF, Moake JL, Nolasco L, Bernardo A, Arceneaux W, Shrimpton CN, Schade AJ, McIntire LV, Fujikawa K and López JA: ADAMTS-13 rapidly cleaves newly secreted ultralarge von Willebrand factor multimers on the endothelial surface under flowing conditions. Blood 100: 4033-4039, 2002.

24. Ley K, Laudanna C, Cybulsky MI and Nourshargh S: Getting to the site of inflammation: The leukocyte adhesion cascade updated. Nat Rev Immunol 7: 678-689, 2007.

25. Reiter RA, Varadi K, Turecek PL, Jilma B and Knöbl P: Changes in ADAMTS13 (von-Willebrand-factor-cleaving protease) activity after induced release of von Willebrand factor during acute systemic inflammation. Thromb Haemost 93: 554-558, 2005.

26. Martin K, Borgel D, Lerolle N, Feys HB, Trinquart L, Vanhoorelbeke K, Deckmyn H, Legendre P, Diehl JL and Baruch D: Decreased ADAMTS-13 (A disintegrin-like and metalloprotease with thrombospondin type 1 repeats) is associated with a poor prognosis in sepsis-induced organ failure. Crit Care Med 35: 2375-2382, 2007

27. Cao WJ, Niiya M, Zheng XW, Shang DZ and Zheng XL: Inflammatory cytokines inhibit ADAMTS13 synthesis in hepatic stellate cells and endothelial cells. J Thromb Haemost 6: 1233-1235, 2008

28. Ono T, Mimuro J, Madoiwa S, Soejima K, Kashiwakura Y, Ishiwata A, Takano K, Ohmori T and Sakata Y: Severe secondary deficiency of von Willebrand factor-cleaving protease (ADAMTS13) in patients with sepsis-induced disseminated intravascular coagulation: Its correlation with development of renal failure. Blood 107: 528-534, 2006.

29. Biron BM, Ayala A and Lomas-Neira JL: Biomarkers for sepsis: What is and what might be? Biomark Insights 10 (Suppl 4): 7-17, 2015.

30. Pepys MB and Hirschfield GM: C-reactive protein: A critical update. J Clin Invest 111: 1805-1812, 2003.

31. Maruna P, Nedelníková K and Gürlich R: Physiology and genetics of procalcitonin. Physiol Res 49 (Suppl 1): S57-S61, 2000.

32. Becker KL, Snider R and Nylen ES: Procalcitonin assay in systemic inflammation, infection, and sepsis: Clinical utility and limitations. Crit Care Med 36: 941-952, 2008.

33. Billeter A, Turina M, Seifert B, Mica L, Stocker R and Keel M: Early serum procalcitonin, interleukin-6, and 24-hour lactate clearance: useful indicators of septic infections in severely traumatized patients. World J Surg 33: 558-566, 2009.

34. Brunkhorst FM, Al-Nawas B, Krummenauer F, Forycki ZF and Shah PM: Procalcitonin, C-reactive protein and APACHE II score for risk evaluation in patients with severe pneumonia. Clin Microbiol Infect 8: 93-100, 2002.

35. Bele N, Darmon M, Coquet I, Feugeas JP, Legriel S, Adaoui N, Schlemmer B and Azoulay E: Diagnostic accuracy of procalcitonin in critically ill immunocompromised patients. BMC Infect Dis 11: 224, 2011.

This work is licensed under a Creative Commons Attribution-NonCommercial-NoDerivatives 4.0 International (CC BY-NC-ND 4.0) License. 